\title{
Full Face Masks Construction and Main Modernization Trends
}

\section{Pavel OTRISAL ${ }^{1,2}$, Stanislav FLORUS ${ }^{1}$, Simona BUNGAU ${ }^{3}$, Sarka HOSKOVA-MAYEROVA ${ }^{4}$}

${ }^{1}$ Nuclear, Biological and Chemical Defence Institute, University of Defence in Brno, Address: Sidliste Vita Nejedleho, 68203 Vyskov, Czech Republic

${ }^{2}$ Faculty of the Physical Culture, Palacký University Olomouc, Address: Trída Miru 117, 77111 Olomouc, Czech Republic

${ }^{3}$ Faculty of Medicine and Pharmacy, University of Oradea, Address: Sq. 1 Decembrie, 10, 410073, Oradea, Romania

${ }^{4}$ Department of Mathematics and Physics, Faculty of Military Technology, University of Defence in Brno, Address: Kounicova 65, 66210 Brno, Czech Republic

E-mails:1 pavel.otrisal@unob.cz; ${ }^{2}$ stanislav.florus@unob.cz; ${ }^{3}$ simonabungau@gmail.com; ${ }^{4}$ sarka.mayerova@unob.cz

\begin{abstract}
In the paper there are described main modernization trends of face masks. Main construction signs of the fourthgeneration masks are introduced in an introductory part. The attention is devoted mainly to masks that can be ordered into a group of the fifth mask generation in further text. A description of development trends is demonstrated on main parts of the facial mask.
\end{abstract}

KEY WORDS: protection mask, inner mask, lens, filter canister, exhalation valve, exhalation valve chamber, inhalation valve, drinking system, speech diaphragm, faceblank, connector, head harness system

\section{Introduction}

Like many products also facial masks are subject to modernization trends aimed primarily at improving the protective properties of the mask as a whole. At present we can talk about the $5^{\text {th }}$ generation of full-face masks. It has to be objectively acknowledged that design trends are mainly determined by military masks, from which the individual components are progressively applied to civilian masks [1,2]. In order to determine the basic modernization trends of the current time, it is necessary to recall the main design characteristics of the $4^{\text {th }}$ generation masks [3-8]. Nowadays, brand new masks should maximally use new technological approaches and must be ready to react on new security threads and be suitable for fulfilment tasks in all kinds of military operations [9-14]. New protection masks should be also tested [16]. In accordance to NATO standards all new established masks should be ready for training in forward [16].

The faceblanks of the $4^{\text {th }}$ generation masks have a shape that adapts to the maximally extent to the shape of the face, minimizing the dead space. Maximum attention is paid to the tightness of full-face masks. Emphasis in this area is dedicated to tightness in the so-called facepiece seal, which is a strip of touching a mask with the user's face. Coupling facepiece seals are used which can be shaped in the problem areas of the face in order to the mask ensures good tightness. The obligatory part of each mask is an inner mask. It is a relatively demanding part to provide a variety of functions. The most important function is to ensure a good flow of inhaled air into the dead space of the mask and to direct the expulsion of exhaled air into the exhaled valve chamber. This is achieved by shaping the inner mask and the inside face of the faceblank. By adjusting the air in the dead space of the mask is achieved the effective visor demisting and reducing the carbon dioxide concentration on the required limit. The inner mask is equipped with check valves for effective airflow. However, increasingly there are also check openings, whose location on the inner mask is more variable than in the case of check valves. The mask is usually provided with one exhalation valve with preload and low value of leakage exhalation valve leakage coefficient. Masks provide users with a good view. This one is ensured by the appropriate shape of the lenses and their location regarding user's eyes and face. The employment of a visor thus one large visor has been gradually growing trend. In order to reduce the stiffness of the mask, the visor was glued to the faceblank, thus avoiding the use of a visor sleeve which, on the contrary, increased the stiffness of the mask. Masks are usually equipped with two 40 x 1/7-inch standard threaded connections, which allow an alternate connection of the filter to the right or left side of the faceblank. The inhalation valve with preload allows the connection to be closed, allowing the filter to be replaced in a contaminated environment without the necessity to leave the contaminated area [17-20]. Speech transmission is usually provided with a speech device that is located in front of the user's mouth or by an auxiliary speech device that replaces a plug of the connector unused to the filter connection. Some masks use both options of speech transmission. The part of the 4th generation masks is a drinking system that allows

\footnotetext{
${ }^{1}$ Corresponding author.

E-mail address: pavel.otrisal@unob.cz.
} 
safe drinking in the contaminated area. A bottle with a special plug is used as a water reservoir allowing safe connection of the bottle with the drinking system device [21,22]. The head harness is designed in order to minimize the pressure of the mask faceblank on the user's head as much as possible. Its most striking feature is a large head plate that is often made of durable mesh. Materials with high breakthrough time and safe for work in explosive or slightly inflammable environments are used for masks' construction. Very chemical and mechanically resistant plastics are used for production of connections, exhalation valve chamber, visor sleeves and other rigid components [23,24]. A typical representative of the 4th generation mask is the British mask FM-12 or, for example, the Finnish mask M-95 (Figure 1).

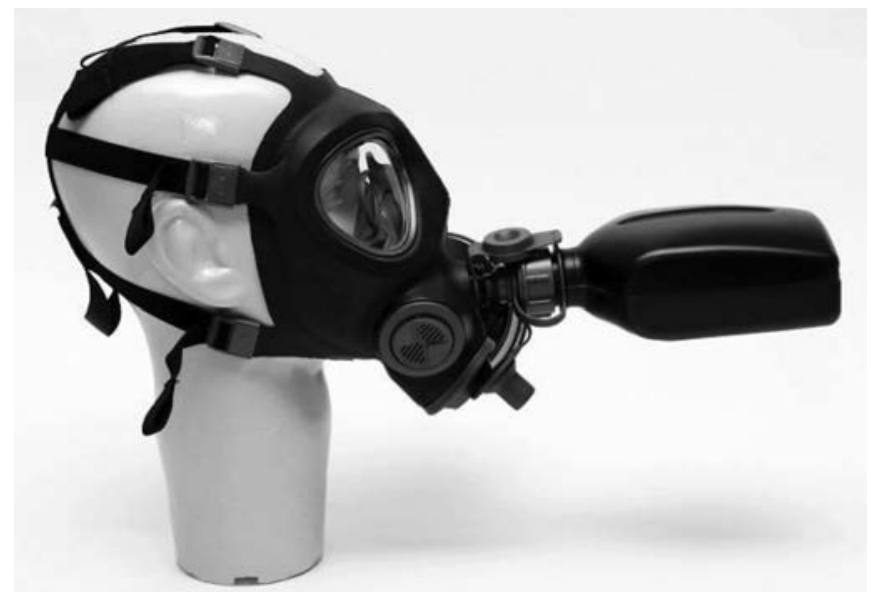

Fig. 1. The full face mask of the 4th generation M-95 with connected drinking system device.

\section{Current Full Face Masks' Construction Trends}

The construction of the 5th generation masks is based, as a matter of principle, on proven design solutions of 4th generation masks. Designation of the mask for certain group of people will influence the shape, construction and selection of the barrier material. However, if the mask is intended to be used primarily for work in a contaminated environment, thus not only for the short-term stay of persons who require protection devices, for example, for evacuation from the contaminated environment, then the faceblank must fulfill relatively demanding structural, protective and physiological requirements. It is assumed that faceblank will be molded from an open mould. The open mold of the faceblank enables quick applying the mask into the protective position. The open mold allows even the employment of relatively rigid construction materials with a high breakthrough time. However, the faceblank stiffness has an impact on the design of the facepiece seal and its shape, especially in a scope of masks for professional departments, which must respect the requirements for the mask functionality as a whole and, in general, ensure compatibility with other material or equipment such as protective helmet, ballistic head protection, body surface protection garments and so forth.

A very problematic issue is the number of faceblanks. A modern trend, especially for masks for the industrial employment, was to produce one size. When working in a highly toxic environment where it is necessary to ensure the maximum face seal on the face, it provides a bigger number of faceblanks with greater guarantee that tightness will be achieved. Experiences from the military environment, which currently represent a relatively large number of women and, in general, people in a broad age range covering the age range of 14 to 60 years, show that the size of the faceblanks or the number of sizes should be given a great deal of attention. Even three sizes of faceblanks currently in use in the Czech Armed Forces do not cover the requirement to secure people with small faces. Although the number of faceblanks sizes is complex, there is a prerequisite that in order to ensure the required mask tightness within a wider professional group, it will be necessary to count with more number of sizes than to make an effort to solve the problem of the tightness with the only one size $[25,26]$. Four sizes appear as an optimum from practical experience. Even in this case, however, the final number of sizes will be determined by the results of the anthropometric survey of the under interest population, the method of designing the faceblank in the facepiece seal, the symmetry of the mask in terms of the weight of the other components (for example the filter), the method of suppression of the possible flapping effect of the filter, system of masks fixation on user's head and so on. The employment of a hood (the whole head faceblank) as a basic constructional (carrier) element of the respiratory protective device of the breathing organs is the certain solution for achieving good tightness. The advantage of using the hood may be on the one hand in the speed of deployment of the device to the protective position, securing the seal in the less problematic area, thus on the user's neck, the possibility of using one size for a wide range of facial sizes, and ensuring tightness even if the user has a beard or tough hair. A similar solution was used, for example, in the Israeli mask for pilots (Figure 2) [27]. 


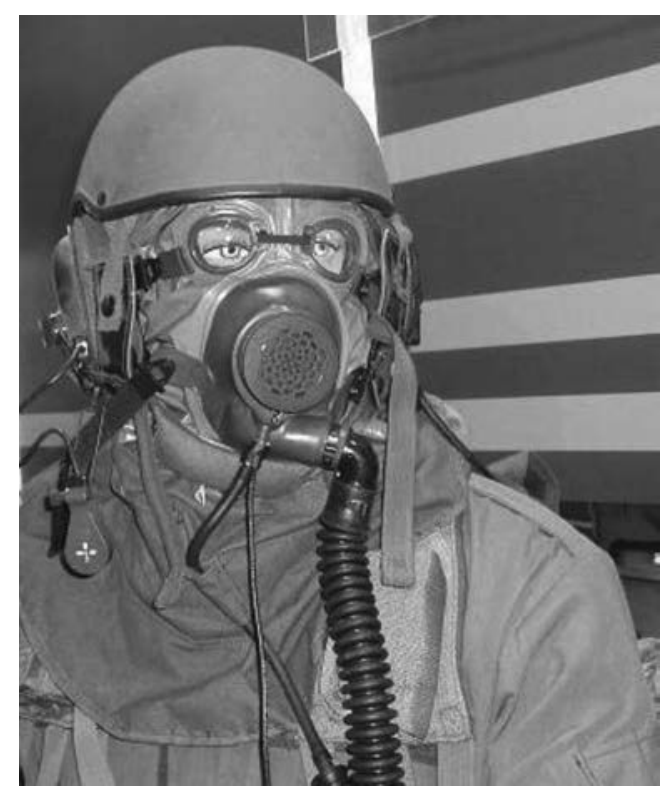

Fig. 2. The Israeli mask for pilots.

The employment of materials that are resistant to suspected contaminants is a necessary condition for longterm stay in contaminated environments. The word of "suspected" needs to be emphasized because there is a general layman's imagination that it is possible to work in a protective garment in any environment for any length of time. Factors that affect the length of stay in the contaminated area are many. The type of used barrier construction material and its chemical resistance to the contaminant is the basis of quality protection. In current time faceblanks are made of bromobutyl-rubber, butyl-rubber, silicone, polyurethane, EPDM, rubber based on natural rubber and so on. It is assumed that similar materials will be used in the future. However, it is also possible to expect different materials to be used on the outside and inside of the faceblank to ensure better dermatological tolerability of the mask. Furthermore, it is not excluded that rigid materials made of highly chemically and mechanically resistant plastics will be used to produce respiratory protective devices that are relatively near to the future. Such a means, however, will most likely constitute integrated protection of the whole head with an internal or external filter-ventilation unit and other elements to ensure communication, bi-directional on-site information and so on. However, it is assumed that similar protective equipment will be designed for a selected range of specialists.

Nowadays, the inner mask is a necessary part of full face masks. Together with the inside face of the faceblank it forms channels for leading exhaled and in filter clean air in the dead space of the faceblank. Inhaled air is thus drawn from the filter through a connection with the inhalation valve to the dead space of the faceblank, where it is directed to the lenses or visor, thereby removing moisture and preventing them from becoming sweating. Then the air passes through the check valves or check holes into the inner mask from which it is inhaled. After exhale, the exhaled air is directed to the inner mask into the exhalation valve chamber, where it overcomes the resistance of the exhalation valve and exits into the external environment. The internal mask plays a decisive role in reducing or maintaining the carbon dioxide content in the dead space below the permissible concentration and also in preventing fogging of the lens or visor. Because the inner mask comes into direct contact with the sensitive parts of the face, it is made of materials that have a minimum negative effect on the skin. Natural rubber or silicone is often used for its production. Other materials that are sufficiently elastic, dermatologically safe, resistant to substances released during breathing, salivation and sweating may be also used. No chemical resistance is required for the suspected contaminants because the inner mask does not come into contact with them. The internal mask is usually detachable to ensure mask maintenance. It is assumed that within newly developed masks, the mask may have a rather complex shape, which will be determined by a requirement for perfect visibility of the lens or visor and for reducing the concentration of carbon dioxide in the dead space. Of course its shape will be influenced by the shape of the faceblank. The emphasis on meeting these requirements can be reflected in the number of inner mask sizes.

A very important part of each full face mask is a facepiece seal. In the scope of the 4th generation masks facepiece seals in the form of sealing cuffs sleeves have been widely used. It should be noted that finding an ideal area for leading the facepiece seal of masks is problematic because the shape of the face may be quite complicated depending on ethnicity or nationality. It is therefore likely that the sealing cuff will also be used in next-generation masks. For example, in the case of Scott's GSR mask, double facepiece seals have been used to ensure the full face mask tightness (Figure 3). One of the least problematic lines is the sealing line on the neck, which is used within hoods. Therefore, it can be assumed 
that this type of sealing line could be used in the future mainly for integrated head protection devices. In the scope of full face masks, however, tightness is achieved by the synergy of the shape of the facepiece seals and faceblank, the size and layout of the tension of the mask head harness system, the location and shape of the filter and so on.

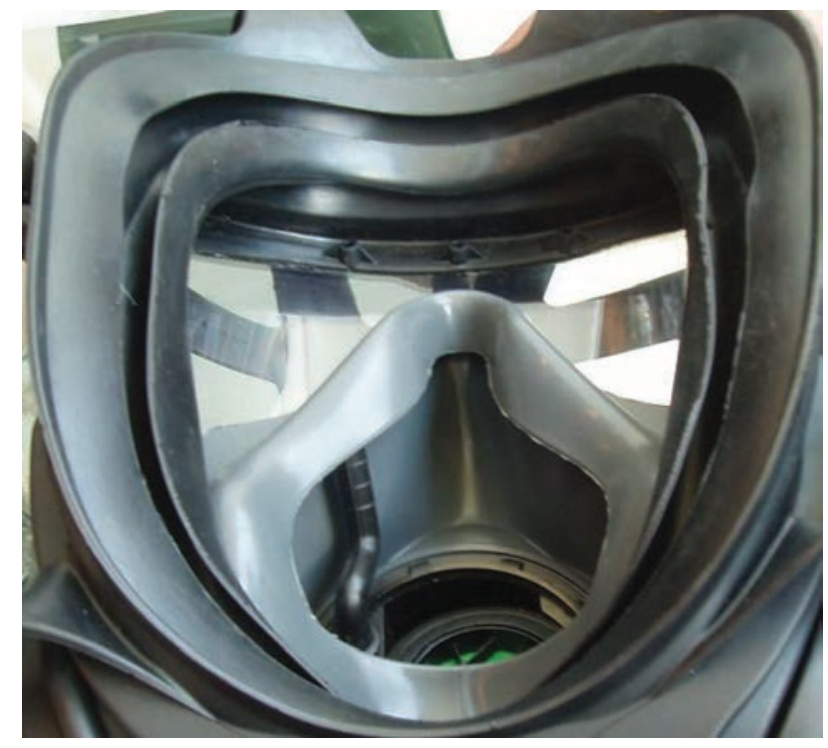

Fig 3. Double facepiece seal of the Scott's GSR mask.

New masks are equipped with two exhalation valves. Just in the 4th generation masks that were introduced into the armament during the 1990s, one of the major modernization trends was the employment of one exhalation valve with preload, which had a short closing time. Although the use of one exhalation valve was less safe for the user than for the two-valve system, the effort to meet lower exhalation resistance requirements led to the use of single-valve systems. When low leakage requirements, which masks with a single valve are not able to meet or meet very difficult, have led to the return to two exhalation valves.

The location of the exhalation valve chamber is solved differently in the scope of contemporary masks. In most cases within the full face masks, however, the exhalation valve chambers are positioned in the front of the mask in front of the user's mouth. Within some masks, the location of the exhalation valve in front of the mouth replaces the function of the speech diaphragm. Masks such as SM-3 or SM-90 (Huber and Suhner, Switzerland), M-95 (Scott, originally Kemira) or OM-90 (Gumárny Zubří, Czech Republic) have an exhalation chamber located in the chin part of the mask. The exhalation valve thus closes the exhaled valve chamber against the direction of gravitation. This location of the valve allows drainage of condensate from the cheek area which is its priority. However, on the tightness of the valve can have a significant effect vibrations caused by user's movement, especially when running, after a jump impact or in an immediate reaction to the external environment (conditions, commands) associated with rapid and automatic head movement. In this case, it can come to the pulsation of shutter valve portion, to impair its tightness and thus to loss of valve protection properties. To the loss of tightness of the valve due to mechanical leakage can then occur even in the case when diffusion of the harmful substance has occurred in the valve material. Within this process, there may be significant changes in mechanical properties of the valve or its shape deformation. Exhalation valves of masks are thus placed in such a position which limits the negative impact of shocks caused by the mask user's movement. The exhalation valves themselves are made of materials that are resistant against chemical warfare agents and military significant toxic industrial chemicals of the organic solvents type. It can be assumed that the exhalation valves will be selectively produced for a certain range of pollutants it means from the chemically resistant material, but which, in terms of flexibility and construction, will meet the requirements for the closure speed and achieve the necessary tightness of the exhalation valve on the valve seat, thus it will provide low leakage values.

The lenses are constructed in dependence on the mask designation. In the scope of masks of the 4th generation, the representation of the lenses, thus the binocular system, represented approximately $60 \%$, and the rest of the masks used visors. Visors are mainly used within newly introduced masks. The advantage of the visor lies mainly in ensuring a good outlook from the mask. The materials of visors, at least within military masks are flexible and are connected with the faceblank. Unfortunately, available information does not indicate whether it is binding with bonding or welding. The visor frames are not thus used because they increase the stiffness of the mask especially in the area of the temples and forehead. The requirement for elasticity of the visor is mainly due to the provision of good tightness in the facepiece seal. The filter connection has undergone relatively significant changes. In the scope of the 4th generation masks, 
one or two 40x1/7" standard thread connections were used, where the number 40 denotes the thread diameter in millimeters and the $1 / 7$ pitch of the lead angle of thread in inches. One connector was located at the front of the mask. Whether the mask had two connectors they were on the right and left side of the mask. There were, however, a number of exceptions. The Swiss SM-3 mask had three connectors (Figure 4), the Belgian BEM-4GP mask had one connector that, allowed the filter to be pulled to the right, left or down.

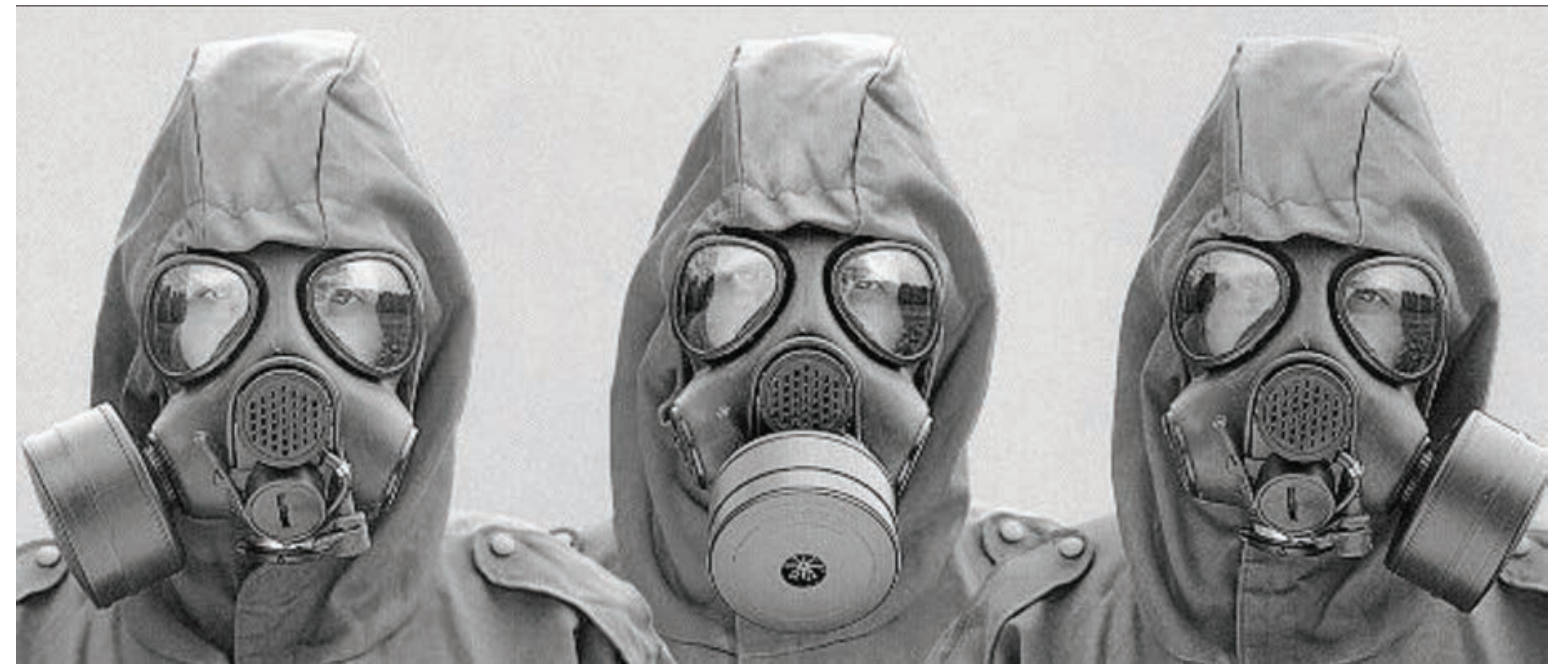

Fig. 4. Swiss SM-3 mask from the HUBER+SUHNER AG company [28].

The trend of the 5th-generation military masks is the employment of connectors for fast connection of the filter - the type of bayonet connector or plug-in connector. The advantage of this type of connection is mainly the speed connection and the tightness of the filter, which, when changing the filter in a contaminated environment, plays a significant role. The tight connection of the filter with the connector is given by the unique position of the filter, which is also secured in the correct position by the locking mechanism. This allows better check of the filter tightness based on checking the correct position of the connected filter. Securing the filter then prevents it from accidentally letting (loose tightness of connection) during shakes associated with the practical use of the mask. The disadvantage of the solution is incompatibility with filters with standardized thread. If the bayonet type is the right step, it will show practice.

The inhalation valve is also the part of the connector. In the case of changing filter in the contaminated area the 4th generation masks' inhalation valves were made of resistant materials and they had generally a preload which enabled the valve to close tightly. It is assumed that when using filters directly attached to the mask, the inhalation valve will continue to have its irreplaceable position as a construction part of the connector. This is also apparent within the 5th generation masks, when the inhalation valve must shut the connector hermetically and when the filter is connected, it must be opened and allowed to flow through the cleaned air to the dead space of the mask. Bayonet connectors, however, allow the employment of different types of inhalation valves or closure devices that can increase the mask user's safety when disconnecting and reconnecting the filter in the contaminated environment.

The filters are also changed a lot. Filter canisters are made of resistant plastics. It can be observed that the shape of the faceblank and the filter canister interact with each other. The center of gravity of the filter canister itself approached the faceblank what significantly eliminated the so-called the flip-flop effect. This occurs as a result of a sharp turn of the head, depending on the speed of its movement and the weight of the filter canister itself, its inertial movement in the direction of the head turning causes the mask to tightness in the facepiece seal on the opposite side of the connected filter canister. Mainly in the scope of military masks, it is possible to observe the use of cartridge filters that are attached to both sides of the mask. The connection is not solved by means of a connection thread but by a bayonet connector as described above. The question being debated is how large the number of harmful substances and which amount are to be captured by the filter. The question is very difficult to answer, especially in the case of toxic industrial chemicals. It is not possible to count with the gas filter universality. It does not exist and cannot be expected even in the future. This is due to known capture mechanisms and their limitations, for example due to the high temperature and nature of potential harmful substances or their possible combinations, which may be theoretically unlimited. It is therefore necessary to accept the fact that filtration protection has its limits that need to be known and used in practice. As a possible solution to extend the filter canister's capability, additional filter canisters can be used to connect to another filter canister. Such a solution is already used in practice.

The head harness system has a decisive influence on the tightness of the mask along the facepiece seal. 
However, the force of the clamping must not cause a physiological discomfort. For this reason, the occipital parts (head plate) of the modern mask head harness systems cover a large area of the head in order to spread the respective pressure on the largest surface. The large occipital port, thanks to the contact surface, will better maintain the mask in the selected head position. To ensure better breathability, the occipital part is usually made of resistant mesh or breathable materials. The head harness system is fastened to the mask by clamping strips and buckles, typically plastic, which allow the clamping force to be adjusted. It is assumed that similar head harness systems will be used for newly developed masks. However, it is possible to assume that for selected groups of specialists, the mask will be clamped with a special catch system to the head protection device, thus creating an integrated protection system. Such systems are already being presented by manufacturers, but they are still more development projects and it is a question of when or whether they will be introduced into mass use.

Drinking system becomes more and more integral with full face masks. Complications in its use in contaminated space due to the need of decontaminated a special bottle plug, the inability to receive fluids at any time of work, concerns about fluid intake in the contaminated area even within keeping all necessary safety procedures and precautions, limited fluid and work and safety requirements when using the individual protective equipment, especially in hot climatic conditions, they find a solution to this problem. Currently, hydration devices allow a firm but dismountable connection to the suction valve of the full face mask drinking system device. The connection is secured against the penetration of the contaminant and is mechanically resistant to breakage. Water dispensers for these devices typically have a volume of 2 to 3 liters, allowing for hydration for 2.5 to 4 hours, while ensuring the most effective physiological water requirement of 0.8 L.h-1. Hydration devices are carried on the back. There are products available on the market where the manufacturer declares their resistance against chemical warfare agents. Although the hydration equipment is designated for use on body surface protection garment, it can also be designed for use under the protective garment of an isolated type. It is a prerequisite that a basic requirement for sufficient fluids supply for securing suitable working conditions when working in protective garments will be ensuring enough intake of fluids which can be difficult to achieve without the use of hydration devices.

Communication is a basic presumption for successful accomplishment of the task. This demand must be also considered by full face masks' designers. Within some 4th generation masks, voice transmission was provided via the exhalation valve. The exhalation valve chamber was placed in front of the mouth. More often, however, sonic devices were used in the form of speech diaphragm located in the front of the mask. A protective mask kit and an auxiliary speech diaphragm were often included in the protective mask kit designed to be screwed into an empty filter connection for better mobile communication. However, technical devices such as voice amplifiers attached from the outside to the speech diaphragm, headsets with microphones or throat microphones have been also used. At the present time voice transmission is provided with a sound system. However, masks are designed for connection of headsets made up of a microphone, headphones and the necessary connecting element to the communication means.

The population has a large proportion of people suffering from various eye defects, which need to be corrected with dioptric glasses. Even manufacturers of protective masks respond to this fact. Each manufacturer adapts the design of the spectacle insert to a particular type of the mask. However, interesting solutions are emerging in the use of some "swimming glasses". These glasses are fully made of polymeric materials which, like swimming glasses, fit on the head. Dioptrical glasses are inserted into the glasses as required by the user. A certain advantage of this solution is that such designed glasses can be worn continuously even without the mounted mask. The disadvantage is the need to put glasses in front of the mask if the user normally uses standard glasses. This prolongs the time for mounting the mask. In addition to glasses, the mask can be equipped with removable shades of different colors to protect eyesight under various lighting conditions or to protect eyes for example against laser radiation. However, shades are usually only included for military use.

It is very often a question of whether it is possible to equip gas filters for individual protection with an indicator of exhaustion in connection with filters. To this date, relatively simple and not very accurate methods have been proposed and implemented. These methods allowed to find up filter exhaustion based on the increase pressure loss of the filter or on the basis of makeweight. Both methods cannot provide information concerning what caused the pressure loss or increase or the weight of the filter. Moreover, these methods are only applicable after using the filter, and even if the two measured values are not limited, the further use of the filter in the contaminated environment would be dangerous for the user of the protective garment itself. However, the rapid development of sensor technologies and their miniaturization, coupled with miniaturization of feed cells, can lead to a reversal even in this area, and the indicator of exhaustion working on a physical principle, accurate enough to indicate the exhaustion of the sorption capacity of the filter for the under interest harmful substances can be implemented in the foreseeable future. 


\section{Conclusion}

The mask of the present time is a highly sophisticated product that has to meet a number of requirements. However, their providing is in many cases a compromise of the required and possible. The constructional solution must be above all based on the determination of the mask itself, from which the requirements for safety, resistivity, and compatibility and so on are unwinded. It should be emphasized that mask quality alone does not guarantee user safety. This is mainly given due to the user's knowledge of the mask's protective properties, his readiness to use the mask, knowledge of the possibilities and limitations of the filter capture and the properties of the contaminant. It is necessary to look at the mask as a protective work aid and, with this in mind, access its acquisition and employment.

\section{Acknowledgements}

This work was conducted within the framework of the NBC Defence Institute of the University of Defence long-term intention of the organization development "Research on methods and technologies of protection against the effects of weapons of mass destruction and industrial hazardous substances" (PROTECT), the project "Development of capabilities and sustainability of logistic support systems" ROZVOLOG, DZRO K-109 and the project for development of basic and applied research developed in the long term by the departments of theoretical and applied bases FMT VÝZKUMFVT, DZRO K-217 supported by the Czech Republic MoD.

\section{References}

1. Svarcova, I., Hoskova-Mayerova S., Navratil, J., Crisis Management and Education in Health, The European Proceedings of Social \& Behavioural Sciences EpSBS, Volume XVI, 2016, p. 255-261. doi:10.15405/ epsbs.2016.11.26.

2. Bogdan, M., Endres, L., Pasca, B., Tit, D., M., Uivarosan, D., Copolovici, D. M., Aleya, L., Bungau, S. Study on the stability and compatibility of the cosmetic products with Lavandula angustifolia oil kept in PPH polypropylene homopolymer plastic containers, Materiale Plastice, 56(1), 2019, p. 133-137.

3. Kellnerová, E., Binková, K., Hošková-Mayerová. Assessment of the efficiency of respiratory protection devices against lead oxide nanoparticles. Models and Theories in Social Systems. Studies in Systems, Decision and Control 179, 2019, p. 257-272. Springer, doi: 10.1007/978-3-030-00084-4_14.

4. Stodola, P. Improvement in the model of cooperative aerial reconnaissance used in the tactical decision support system. Journal of Defense Modeling and Simulation, 14(4), 2017, p. 483-492.

5. Štěpánek, B., Otřísal, P. The Development and Establishment Process of Centres of Excellence in North Atlantic Organization. Croatian Journal of Education-Hrvatski Casopis za Odgoj i obrazovanje, 14(1), 2012, p. 169-174.

6. Otř́sal, P., Hošková-Mayerová, Š. Selected Aspects of Barrier Materials Assessment as a Part of the Reaction on Threats and Risks Connected with CBRN Problems. Decision Making in Social Sciences: Between Traditions and Innovations 247. Ed. Kacprzyk, J., Springer, 2020, p. 531-543.doi: 10.1007/978-3-030-30659-5_32.

7. Tamuliene, J., Sarlauskas, J., Bekesiene, S. Modeling and investigation of new explosive materials based on N-(3,5-dimethyl-2,4,6-trinitrophenyl)-1H-1,2,4-triazol-3-amine, Journal of Molecular Modeling, 23(8), 2017, art. no. 228, doi: 10.1007/s00894-017-3399-4.

8. Beňová, P., Hošková-Mayerová, Š, Navrátil, J. Terrorist Attacks on Selected Soft Targets. Journal of Security and Sustainability Issues, 2019, 8(3), p.453-471. 10.9770/jssi.2019.8.3(13)

9. Otř́sal, P. Decontamination Modules Formed by the Czech Armed Forces Chemical Corps. Croatian Journal of Education-Hrvatski Casopis za Odgoj i obrazovanje, 14(1), 2012, p. 123-127.

10. Otrisal, P., Obsel, V., Buk, J., Svorc, L. Preparation of Filtration Sorptive Materials from Nanofibers, Bicofibers, and Textile Adsorbents without Binders Employment. Nanomaterials, 8(8), 2018, p. 564. https://doi.org/10.3390/ nano8080564.

11. Prikryl, R., Otrisal, P., Obsel, V., Svorc, L., Karkalic, R., Buk, J. Protective Properties of a Microstructure Composed of Barrier Nanostructured Organics and SiOx Layers Deposited on a Polymer Matrix. Nanomaterials, 8(9), 2018, p. 679. https://doi.org/10.3390/nano8090679.

12. Bekesiene, S., Hoskova-Mayerova, S., Becherova, O. Accidents and emergency events in railway transport while transporting dangerous items, In: Proceedings of 20th International Scientific Conference. Transport Means. Kaunas:Kaunas University of Technology, 2016, p. 936-941.

13. Talhofer, V., Hošková-Mayerová, Š. Method of Selecting a Decontamination Site Deployment for Chemical Accident Consequences Elimination: Application of Multi-Criterial Analysis. ISPRS Int. J. Geo-Inf., 2019, 8(4), p. 22. doi:10.3390/ijgi8040171. 
14. Florus, S., Otřísal, P. Selected methods of study of chemical resistance of insulation protective films for chemical warfare agents. Chem. Listy, 108(9), 2014, p. 838-842.

15. Plzakova, L., Krocova, Z., Kubelkova, K., Macela, A. Entry of Francisella tularensis into murine B cells: The role of B cell receptors and complement receptors. PLoS ONE, 10(7), 2015. Article Number: e0132571.

16. Hoskova-Mayerova S. Education and Training in Crisis Management, The European Proceedings of Social \& Behavioural Sciences EpSBS, Volume XVI, (2016) p. 849-856. doi:10.15405/epsbs.2016.11.87.

17. Otřísal, P., Florus, S. Current and perspectives in personal and collective protection against effects of toxic compounds. Chem. Listy, 108(12), 2014, p. 1168-1171.

18. Glevitzky, I., Dumitrel, G.A., Glevitzky, M., Pasca, B., Otrisal, P., Bungau, S., Cioca, G., Pantis, C., Popa, M. Statistical analysis of the relationship between antioxidant activity and the structure of flavonoid compounds, Rev. Chim.-Bucharest, 70(9), 2019, 3103-7.

19. Hošková-Mayerová, Š. Emergency assessment in case of hazardous substance leakage at Czech Republic freight rail transport in 2008-2016. In: Safety and Reliability - Safe Societies in a Changing World, Proceedings of ESREL 2018, London: CRC Press, 2018, p. 1381-1386, doi: 10.1201/9781351174664-174.

20. Endres, L., Tit, D., M., Bungau, S., Cioca, G., Abdel-Daim, M., Buhas, C., Pop, O., Sava, C. Markers usefulness in the melanic metastatic celular epitops identification in the sentinel lymph node, Rev. Chim.-Bucharest, 69(12), 2018 , p. 3675-3679.

21. 83 2204.Respiratory protective devices. List of equivalent terms. (in Czech). May 1994. Praha: Český normalizační institut, 1993.

22. 83 2203. Respiratory protective devices. Nomenclature of components. (in Czech). January 1994. Praha: Český normalizační institute, 1993.

23. Florus, S. Military protection masks of the 4th generation of the European countries. (in Czech) [University textbooks]. Vyškov : VVŠ PV, 1999. 96 p.

24. Florus, S. Actual modernization trends in the protective masks construction. (in Czech). Conference proceedings VVŠ PV. Vyškov, 1999, No. 2, p. 129-138.

25. Navrátil J., Sadovská V., Švarcová I. Health Risk Assessment of Combustion Products from Simulated Residential Fire. Ed. Hošková-Mayerová Š., Maturo F., Kacprzyk J., Mathematical-Statistical Models and Qualitative Theories for Economic and Social Sciences. Studies in Systems, Decision and Control 104. p.15-23, Springer, Cham, 2017 doi.org/10.1007/978-3-319-54819-7_2.

26. Bungau, S., G., Abdel-Daim, M., M., Tit, D., M., Ghanem, E., Sato, S., Maruyama-Inoue, M., Yamane, S., Kadonosono, K. Health benefits of polyphenols and carotenoids in age-related eye diseases, Oxidative Medicine and Cellular Longevity, 2019, ID 9783429. https://doi.org/10.1155/2019/9783429

27. Florus, S., Otřísal, P. Construction of facial masks. (in Czech). Brno: Tribun EU s. r. o., 2015, 162 p. ISBN $978-$ 80-263-0881-2.

28. HUBER+SUHNER AG, Pfäfikon : Protection Mask SM3/SM90. 1994. 11 p. 